Proc. Nat. Acad. Sci. USA

Vol. 68, No. 9, pp. 2102-2107, September 1971

\title{
Fluctuations in Nonequilibrium Systems
}

(chemical kinetics/birth and death model/phase-space description/Langevin/Volterra-Lotka)

\section{G. NICOLIS AND I. PRIGOGINE*}

Faculté des Sciences, Université Libre de Bruxelles, Brussels, Belgium

Contributed by I. Prigogine, June 25, 1971

\begin{abstract}
The theory of fluctuations is extended to nonlinear systems far from equilibrium. Systems whose evolution involves two separate time scales, e.g., chemically reacting mixtures near a local equilibrium regime, are studied in detail. It is shown that the usual stochastic description of chemical kinetics based on a "birth and death" model is inadequate and has to be replaced by a more detailed phase-space description. This enables one to develop for such systems a plausible mechanism for the emergence of instabilities, in which the departure from the steady state is governed by large fluctuations of macroscopic size, while small thermal fluctuations are still described by a generalization of Einstein's equilibrium theory. On the other hand, far from a local equilibrium regime, infinitesimal fuctuations may increase and attain macroscopic values. In this case the system evolves to a state of "generalized turbulence", in which the distinction between macroscopic averages and fluctuations becomes meaningless.
\end{abstract}

Certain types of nonlinear systems maintained beyond a critical distance from thermodynamic equilibrium may undergo instabilities in their steady-state solutions. Hydrodynamic transitions such as thermal convection are well known examples of this behavior. Purely dissipative systems such as chemically reacting mixtures may also present instabilities and evolve subsequently to new regimes showing spatial, temporal, or functional organization (1).

It is important to distinguish between "macroscopic" transitions and phenomena such as plasma instabilities (2) or laser thresholds (3). The origin of the latter is "molecular" in the sense that the momentum distribution function has a highly non-Maxwellian form. As a result, the system becomes unstable with respect to infinitesimal thermal fluctuations and may exhibit a critical fluctuation behavior in the neighborhood of the onset of the instability. In contrast, macroscopic transitions in chemical kinetics and fluid dynamics occur, as a rule, under conditions that are close to a local equilibrium state. Yet these systems, which locally remain dissipative, and thus tend to damp thermal fluctuations, undergo large-scale transitions that change their macroscopic state (appearance of convection cells, spatial dissipative structures, and limit cycles in chemical autocatalytic systems, etc.).

* Also at the Center for Statistical Mechanics and Thermodynamics, University of Texas, Austin, Texas.
An understanding of the molecular origin of such organization phenomena requires a detailed study of fluctuations around the nonequilibrium state that is going to become unstable. The present paper outlines an approach to this problem based on the theory of stochastic processes (4).

The theory of fluctuations has been developed extensively for systems near thermodynamic equilibrium. The classical approach is based on a combination of the ideas of linear thermodynamics of irreversible processes and of equilibrium considerations (5). Equivalent to this formulation is the Langevin method (6), which amounts to introducing $a$ priori the fluctuations in the equations of evolution through suitably correlated stochastic force terms. A potentially more powerful method consists in the derivation of stochastic master equations based on the assumption that the kinetic equations define a markovian process. As a rule, these equations represent a birth and death process (7) $\dagger$. A still more general approach is based on statistical mechanics, with a kinetic equation such as the Boltzmann equation as a starting point (8).

An important result, recently proved in full generality (9), establishes that around equilibrium all the methods lead to equivalent results. In particular, the one-time fluctuations around equilibrium are shown to satisfy in the limit of small fluctuations the well-known Einstein distribution $(5,9)$. Thus, in an ideal reacting mixture, the relative mean quadratic fluctuations $\left(\bar{\delta}^{2} / X_{\theta}^{2}\right)^{1 / 2}, X_{e}$ being the equilibrium average, are very small, of the order of $X_{\mathrm{e}}^{-1 / 2}$. In other terms for such systems there exist clearcut distinctions between macroscopic averages and fluctuations.

The behavior of fluctuations around steady nonequilibrium states is much less known (10). It is only in the domain of linear systems that general conclusions have been derived. Most of the models studied explicitly refer to current fluctuations (11) or to com-

$\dagger$ The first application of stochastic theory to nonlinear chemical kinetics seems to be due to Delbrück (1940). Eigen has applied stochastic theory to the problem of self-organization and evolution of biological macromolecules [Eigen, M., Naturwissenschaften, in press]. 
position fluctuations in open, chemically reacting mixtures (12). In all cases, the equivalence of the Langevin, birth and death, and kinetic equation approach is confirmed. Moreover, small fluctuations are described by the following generalization of Einstein's formula (12):

$$
P(\{\delta X\}) \propto \exp \left[\frac{\left(\delta^{2} S\right)_{0}}{2 k}\right]
$$

with

$$
\overline{\delta X^{2}}=\overline{\bar{X}}=X_{0} .
$$

$\left\{\delta X \equiv X-X_{0}\right\}$ denotes the fluctuation of a set of state variables around the steady state, $k$ is Boltzmann's constant, and ${ }^{1} / 2\left(\delta^{2} S\right)_{0}$ is the second-order excess entropy evaluated around the nonequilibrium stateł.

As we saw above, in order to understand the onset of a macroscopic transition, it is necessary to study fluctuations in nonlinear systems very far from thermodynamic equilibrium. In this domain the validity of the Langevin type of approach is no longer guaranteed (7). This leaves us with the master equations based on birth and death processes and with the more general kinetic equation approach. In Sec. $\mathscr{Q}$ the birth and death process approach is applied to a simple nonlinear chemical kinetic model. $\$$ It is found that this formulation leads to results which, far from equilibrium, are different from (1.1). On the other hand, a number of general arguments (see Sec. 2) indicate that in the whole range of local equilibrium theory in which local thermodynamic variables may be used to describe irreversible processes $(1,4)$, Eq. (1.1) should remain valid for small fluctuations. In Sec. 3, we show that in order to express consistently the local equilibrium condition it is necessary to adopt a phase-space description based on a Boltzmann-type kinetic equation. Assuming that this equation defines a markovian process in the complete phase space, we then show that Eq. (1.1) is recovered in the limit of small fluctuations. This unexpected result implies that for nonlinear systems far from equilibrium, the usual birth and death stochastic approach is generally inadequate. Sec. $4-6$ are devoted to the study of oscillatory systems without asymptotic stability. In Sec. 4, the Volterra-Lotka model is considered in its chemical kinetic and ecological versions. We show that the usual stochastic analysis predicts that the steady state is unstable with respect to infinitesimal fluctuations. On the other hand, the phase-space approach, outlined in Sec. 5, predicts that small thermal fluctuations are stable and obey Eq. (1.1). In Sec. 6, the implications of these results in chemical kinetics and ecology are discussed. An analogy is drawn between the

$\ddagger$ The models treated in refs. $10-12$ are all stable with respect to arbitrary perturbations.

$\S$ Chemical kinetic models are chosen due to the simplicity of the formalism (the stochastic variables are integers) and since these models may give rise to oscillatory behavior and to instabilities far from equilibrium. behavior of fluctuations, which in the Volterra-Lotka model attain a macroscopic level, and the well-known phenomenon of turbulence in fluid dynamics. A plausible mechanism of setting up a macroscopic instability in a dissipative system is also outlined.

\section{A SIMPLE NONLINEAR MODEL}

The main ideas of this Section will be illustrated on the simple bimolecular scheme

$$
\begin{gathered}
\mathrm{A}+\underset{\mathrm{M}}{\stackrel{k_{1}}{\rightarrow}} \mathrm{X}+\mathrm{M} \\
2 \mathrm{X} \stackrel{\mathrm{m}}{\rightarrow} \mathrm{E}+\mathrm{D} .
\end{gathered}
$$

A slightly different version of this scheme was considered by Babloyantz and Nicolis (12). A comparison between their results and the conclusions of this section is given in ref. 4. The system is open to large reservoirs of $\mathrm{A}, \mathrm{M}, \mathrm{D}, \mathrm{E}$. The inverse reaction rates are neglected: the system thus operates automatically far from thermodynamic equilibrium.

Assuming the reaction occurs in ideal mixture conditions and that the system remains spatially homogenous, one can write the usual conservation of mass equations which determine the time evolution of $X$. These equations admit a single steady-state

$$
X_{0}=\left(k_{1} A M / 2 k_{2}\right)^{1 / 2}
$$

that is asymptotically stable with respect to arbitrary perturbations.

In order to study the fluctuations around state (2.2), we make the usual assumption that the state of the system is described in terms of a probability function $P(A, M, D, E, X, t)$. The evolution of this function is given in terms of a birth and death type of master equation $(4,12)$, which in reduced form (summed over all reservoir variables) reads:

$$
\begin{aligned}
\frac{d P(X, t)}{d t}= & k_{1} \bar{A} \bar{M} P(X-1, t)-k_{1} \bar{A} \bar{M} P(X, t) \\
& +k_{2}(X+1)(X+2) P(X+2, t) \\
& -k_{2} X(X-1) P(X, t) .
\end{aligned}
$$

$\bar{A}, \bar{M}$ are averages over the reservoir states (i.e., known quantities) and are seen to appear as parameters.

Eq. (2.3) admits a steady-state solution, which can be computed exactly in the thermodynamic limit

$$
X_{0} \rightarrow \infty, V \rightarrow \infty,\left(X_{0} / V\right)=\text { finite }
$$

where $V$ is the volume of the system. The mean quadratic fluctuation corresponding to this distribution can easily be computed (4). The result is a value different from the Poisson variance:

$$
\overline{\delta X}^{2}=\frac{3}{4} \bar{X} \simeq \frac{3}{4} X_{0}
$$

The steady-state solution is thus incompatible with the generalized Einstein form (1.1), even in the limit of 
small fluctuations. The factor $3 / 4$ in front of $X_{0}$ in Eq. (2.5) is model-dependent. It appears, therefore, that for nonlinear systems the stochastic master equations do not admit solutions of a universal form, but rather they predict a behavior for the fluctuations that depends strongly on the detailed kinetic properties of the reaction.

This conclusion cannot be valid in gen rral. Let us recall that in chemical kinetics one usually deals with systems described by a local equilibrium theory $(1,4)$, in which the state functions (entropy, density, etc.) are described, locally, by the same independent variables as in equilibrium. It is natural to expect that in this case Eq. (1.1) should also apply. A sufficient condition for the validity of this theory is that the distribution of momenta deviates only slightly from a local Maxwellian form (1). T However, this implies the existence of two largely separated time scales (4): a short relaxation time between frequent elastic collisions, which restore continuously an average Maxwellian distribution, and a longer, macroscopic scale over which the chemical composition changes as a result of reactive collisions that tend to perturb the Maxwellian distribution. The effects related to the relaxation time cannot be accounted for in a description of the type outlined in this section, where the internal states (e.g., the values of the momenta) of the molecules are discarded. Thus, fluctuations must be discussed on the basis of a kinetic equation, such as the Boltzmann equation, containing the effect of both reactive and elastic collisions.

\section{PHASE-SPACE DESCRIPTION}

Let us now illustrate briefly the phase-space theory of fluctuations in the simple example considered in Sec. 2 . As we deal with dilute mixtures, the starting point of the average description will be the Boltzmann equation. We adopt the notation $\bar{F}_{\alpha}^{\gamma}$ for the Boltzmann probability density of component $\gamma$ corresponding to an internal state $\alpha$, and assume for simplicity that the spectrum of $\alpha$ is discrete. The bar over $F_{\alpha}{ }^{\gamma}$ indicates that in the Boltzmann equation, $\bar{F}_{\alpha}^{\gamma}$ represents an average quantity.

The Boltzmann equation corresponding to model (2.1) reads (13)

$\frac{d \bar{F}_{\alpha}{ }^{X}}{d t}=\sum_{j k l} B_{j k \alpha l} \bar{F}_{j}^{A} \bar{F}_{k}{ }^{M}-2 \sum A_{\alpha j k l} \bar{F}_{\alpha}{ }^{X} \bar{F}_{j}{ }^{X}+\left(\frac{d \bar{F}_{\alpha}{ }^{X}}{d t}\right)_{e l}$.

In the right of this equation, $\left(d \bar{F}_{\alpha} x / d t\right)_{e l}$ describes the effect of elastic collisions; the remaining two terms refer to reactive collisions. $B_{i j k l}$ and $A_{i j k l}$ are the transition probabilities per unit time for scattering between 2

T This does not exclude the existence of large overall constraints such as chemical affinities that may drive the system very far from thermodynamic equilibrium. molecules in states $(k l)$ into 2 molecules in states $(i j)$ for the reactions corresponding to the two steps in (2.1). They satisfy the well-known conditions:

$$
\begin{aligned}
& A_{i j, k k}=0 \\
& A_{i j, k l}=A_{j i, k l}=A_{i j, l k} \geq 0 \text { for }(k l) \neq(i j) \\
& \sum_{k l} A_{i j, k l}=0 .
\end{aligned}
$$

According to the remarks made in the previous section, at the limit of very frequent elastic collisions we obtain:

$$
\bar{F}_{\alpha}^{x} \simeq \bar{F}_{\alpha, 0}^{x, 0} \text { local Maxwellian }
$$

and similarly for A, B, D, E, i.e.

$$
\left(d_{t} \bar{F}_{\alpha}^{x}\right)_{e l} \simeq 0 .
$$

We may thus neglect the explicit effect of elastic collisions in the Boltzmann equation. Of course, the influence of these collisions remains implicitly in the reactive terms through the fact that the molecular speed distributions are now Maxwellian and that distinction is made between molecules occupying different momentum states.

Let us set $\bar{F}_{\alpha} \Delta r \Delta p=\bar{f}_{\alpha}, \bar{f}_{\alpha}$ being the average number of molecules in the phase-space volume $\Delta r \Delta p$. To describe the fluctuations around this average occupation number, we assume that Eq. (3.1) defines a Markovian process in the complete phase space. It is then easy to derive, by a method due to Siegert (14), a master equation for the probability function $P(\{f\}, t)$. The reduced form of this equation, summed over all reservoir variables, reads (4):

$$
\begin{aligned}
& \frac{d P\left(\left\{f^{X}\right\}, t\right)}{d t}=\sum_{i j k l} B_{i j k l} \bar{f}_{i}^{A} \bar{f}_{j}{ }^{M} \\
& \quad \times\left[P\left(f_{k}{ }^{X}-1,\left\{f^{\prime}\right\}, t\right)-P\left(f_{k}{ }^{X},\left\{f^{\prime}\right\}, t\right)\right] \\
& +\sum_{i j k l} A_{i j k l}\left[( f _ { i } { } ^ { X } + 1 ) ( f _ { j } { } ^ { X } + 1 ) P \left(f_{i}{ }^{X}+1, f_{j}{ }^{X}+1\right.\right. \\
& \left.f_{k}{ }^{X}-1, f_{l}{ }^{X}-1,\left\{f^{\prime}\right\}, t\right) \\
& -f_{i}{ }^{X} f_{j}{ }^{X} P\left(f_{i}{ }^{X}, f_{j}{ }^{X}, f_{k}{ }^{X} f_{l}{ }^{X},\left\{f^{\prime}\right\}, t\right) .
\end{aligned}
$$

$\left\{f^{\prime}\right\}$ denotes the occupation numbers of the states not implied in the reaction steps. When Eq. (3.4) is multiplied by $f_{\alpha}{ }^{x}$ and averaged over all $f_{\mathrm{s}}$, it yields Eq. (3.1), provided a factorization assumption is made on $P$.

The difficulty in solving Eq. (3.4) arises from the infinite number of coupled terms contained in the sums over internal states on the right side. For this reason, we study this equation in the limit of small fluctuations. One obtains a Fokker-Planck-type equation satisfied by the reduced probability functions for individual level occupations. 
The final result reads (4):

$$
\begin{aligned}
\frac{\partial P_{1}\left(x_{\alpha}, t\right)}{\partial t}=2\left(\sum_{j k l} A_{\alpha j k l} \bar{f}_{j}^{X}\right) \frac{\partial}{\partial x_{\alpha}} x_{\alpha} P_{1}\left(x_{\alpha}, t\right) \\
+\frac{\partial^{2} P_{1}\left(x_{\alpha}, t\right)}{\partial x_{\alpha}{ }^{2}} 2 \tilde{f}_{\alpha}^{X} \sum_{j k l} A_{\alpha j k l} \tilde{f}_{j}^{X}
\end{aligned}
$$

We have set

$$
f_{\alpha}{ }^{x}=\bar{f}_{\alpha}{ }^{x}+x_{\alpha}
$$

At the steady state, this equation reduces to a form that no longer contains explicitly the transition probabilities $A_{i j k l}$ and $B_{i j k l}$, and which admits the solution:

$$
P_{1}\left(x_{\alpha}\right)=\left(2 \pi \tilde{f}_{\alpha}{ }^{x}\right)^{-1 / 2} \exp \left[-\frac{x_{\alpha}{ }^{2}}{2 f_{\alpha}{ }^{x}}\right]
$$

for which

$$
\overline{x_{\alpha}{ }^{2}}=\overline{\delta f}_{\alpha}^{2}=\bar{f}_{\alpha}{ }^{x}
$$

Integrating this relation over positions and momenta and recalling that $\bar{F}^{x}$ remains locally Maxwellian, we recover the extended Einstein relation (1.1). We see, therefore, that the phase-space description gives rise to results that for small thermal fluctuations assume a universal form compatible with the extended Einstein formula, substantiating the qualitative arguments advanced in the previous section. Comparing the two master Eq. (2.3) and (3.4), one can see that the inadequacies of the usual birth and death type stochastic formulation are due to the fact that in this method the internal states of the system are treated incorrectly. For instance, Eq. (2.3) implies that there is a finite probability that two molecules of $\mathbf{X}$ [see term containing $P(X+2, t)]$ be at the same state. In the more complete phase-space description, the first of the conditions (3.2) implies that, in the thermodynamic limit, the probability of this event is vanishingly small. Necessarily then, a bimolecular step introduces in the general master equation (3.4) 2 molecules of $X$ belonging to two different internal states.

In the more general case of systems far 'rom local equilibrium, although Eq. (3.8) is not expected to be valid, there is no reason to believe that the birth and death process description will again become adequate. It is only for systems that do not admit a statistical mechanical formulation (e.g. social or ecological problems) that this description can be applied, although again there can be no objective criteria assuring that the process is Markovian.

\section{THE VOLTERRA-LOTKA MODEL}

In the model discussed in the previous two sections, the macroscopic steady, state $X_{0}$ or $\bar{f}_{\alpha}{ }^{x}$ was asymptotically stable with respect to arbitrary perturbations. As a preliminary to the problem of fluctuations around nonequilibrium states in the neighborhood of instabilities, we now study a system whose steady state lacks asymptotic stability. The particular model we choose is the
Volterra-Lotka model, which originally was conceived to describe the competition between a number of predator and prey biological species (15).

Let $X, Y$ denote the populations of two interacting predator-prey species. The Volterra-Lotka equations read:

$$
\begin{aligned}
& \frac{d X}{d t}=\epsilon_{1} X-k_{2} X Y \\
& \frac{d Y}{d t}=k_{2} X Y-\epsilon_{3} Y
\end{aligned}
$$

where $\epsilon_{1}, \epsilon_{3}, k_{2}$ are positive. Let us set

$$
\begin{aligned}
& \epsilon_{1}=k_{1} A \\
& \epsilon_{3}=k_{3} D
\end{aligned}
$$

Eq. (4.1) are then the conservation of mass equations of the following set of irreversible autocatalytic chemical reactions (in the limit of an ideal mixture):

$$
\begin{aligned}
& \mathrm{A}+\mathrm{X} \stackrel{k_{1}}{\rightarrow} 2 \mathrm{X} \\
& \mathrm{X}+\mathrm{Y} \stackrel{k_{2}}{\rightarrow} 2 \mathrm{Y} \\
& \mathrm{Y}+\mathrm{D} \stackrel{k_{3}}{\rightarrow} \mathrm{E}+\mathrm{D} .
\end{aligned}
$$

This chemical analog of the Volterra-Lotka model is very useful in understanding the mechanism of fluctuations in the vicinity of the onset of oscillations and instabilities.

The macroscopic behavior of system (4.1) to (4.3) has been analyzed by Volterra (15). The main results are as follows:

(i) The system admits one nontrivial steady-state solution

$$
X_{0}=\frac{k_{3} D}{k_{2}}, \quad Y_{0}=\frac{k_{1} A}{k_{2}} .
$$

(ii) Small perturbations around $\left(X_{0}, Y_{0}\right)$ exhibit undamped oscillations with a universal frequency

$$
\omega_{0}=\left(k_{1} k_{3} A D\right)^{1 / 2}
$$

(iii) For arbitrary perturbations, Eq. (4.1) admit a constant of motion $(15,16)$. Thus, finite perturbations are also periodic in time with periods depending on the initial conditions. The trajectories of the system in the space $(X, Y)$ consist of a dense net of closed curves that are all orbitally stable (but not asymptotically stable).

(iv) Oscillations can only occur in the limit when the system is displaced far from the state of thermodynamic equilibrium $(1,17)$.

We now study fluctuations around state (4.4) or around a periodic trajectory. We first assume, as in Sec. 2 , that the macroscopic equations (4.1) define a birth and death process in $(X, Y)$ space. The master equation for the reduced probability distribution 
$P(X, Y, t)$ reads $(4)$ :

$$
\begin{gathered}
\frac{d P(X, Y, t)}{d t}=A(X-1) P(X-1, Y, t) \\
-A X P(X, Y, t)+(X+1)(Y-1) P(X+1, Y-1, t) \\
-X Y P(X, Y, t)+D(Y+1) P(X, Y+1, t) \\
-D Y P(X, Y, t)
\end{gathered}
$$

A detailed analysis of this equation (4) shows that, as for the model examined in Sec. 2 , the distribution of fluctuations is not given by the Poisson law. Thus, small fluctuations contradict Eq. (1.1). Moreover, the distribution function is always time-dependent and the mean square correlations $\overline{\delta X^{2}}, \overline{\delta Y^{2}}$ are slowly-increasing functions of time, even for infinitesimal fluctuations. Stochastically, therefore, the steady state (4.4) is unstable and the system exhibits, for long times, abnormal critical fluctuations. Ecologically, this situation has a clear interpretation: the steady-state prey distribution is never stable because there is no internal mechanism that reestablishes equilibrium, once the latter is perturbed by the predator.

\section{PHASE-SPACE DESCRIPTION OF THE VOLTERRA-LOTKA MODEL}

The chemical analog (4.3) of the Volterra-Lotka model admits a phase-space description which, according to the results of Secs. 2 and 3 , is the only correct description to be adopted for chemical kinetics in the local equilibrium regime. Assuming again that the system is maintained uniform in space, one obtains, for a dilute mixture, two coupled Boltzmann equations for the average number densities of constituents $\mathrm{X}$ and $\mathrm{Y}$ in a given internal state $(4,13)$ :

$$
\begin{aligned}
\frac{d \bar{F}_{\alpha}{ }^{X}}{d t}=-\sum_{j k l} A_{\alpha j k l} \bar{F}_{\alpha}{ }^{X} \bar{F}_{j}{ }^{A}+ & 2 \sum_{j k l} A_{j k \alpha l} \bar{F}_{j}{ }^{X} \bar{F}_{k}{ }^{A} \\
& -\sum_{j k l} B_{\alpha j k l} \bar{F}_{\alpha}{ }^{X} \bar{F}_{j}{ }^{Y} \\
\frac{d \bar{F}_{\beta}{ }^{Y}}{d t}=-\sum_{j k l} B_{\beta j k l} \bar{F}_{j}{ }^{X} \bar{F}_{\beta}{ }^{Y}+ & 2 \sum_{j k l} B_{j k \beta l} \bar{F}_{j}{ }^{X} \bar{F}_{k}{ }^{Y} \\
& -\sum_{j l k} C_{\beta j k l} \bar{F}_{\beta}{ }^{Y} \bar{F}_{j}{ }^{D} .
\end{aligned}
$$

The transition probabilities per unit time for reactive collisions $A_{i j k l}, B_{i j k l}, C_{i j k l}$ satisfy conditions (3.2). Elastic collisions terms have not been written explicitly, on the assumption that the system has attained a local equilibrium regime.

We observe that the structure of Eq. (5.1) is quite different from Eq. (4.1) or their generalization to many components $(15,16)$ in spite of the fact that, on averaging Eq. (5.1) over internal states, one obtains (4.1). In particular, (5.1) cannot give rise to a constant of motion as in the Volterra (15) or Kerner (16) analysis.

Before we proceed to the study of fluctuations, we wish to emphasize that the two levels of description given by Eq. (4.1) and (5.1) correspond to two largely different, but interesting, ecological situations. In the system described by Eq. (4.1), it is assumed that the individuals of the prey population are consumed indifferently by the predator. When the predator population is comparable to or larger than the prey, it is reasonable to expect that this is indeed the most probable situation. However, in the more realistic case of small predator versus prey ratio, the most natural competition consumes preferentially those prey individuals having small values of some "fitness" parameter that measures the ability to resist to or escape from the predator. It is then natural to expect that in such systems, in addition to the effect of the predators, the internal processes determining the fitness distribution within the prey species should play an important role. This situation is well described by a set of equations of the type (5.1), provided one reinterprets suitably the parameter $\alpha$ determining the internal state. One of the consequences of these processes is to permit an evolution of the prey species in which the unfit individuals are eliminated continuously.

Let us now study the fluctuations around the steadystate solution of Eq. (5.1), and assume that the latter define a Markovian process in the complete phase space. Using the same method as in Sec. 3, one can derive a master equation for the reduced probability distribution $P\left(\left\{f^{X}\right\},\left\{f^{Y}\right\}, t\right)$. In the limit of small fluctuations this gives rise to two Fokker-Planck equations of the form (4):

$$
\begin{aligned}
& \frac{\partial P_{1}\left(x_{\alpha}, t\right)}{\partial t}=\frac{\partial}{\partial x_{\alpha}} x_{\alpha} P_{1}\left(x_{\alpha}, t\right) \\
& \times\left[2 \frac{1}{\bar{f}_{\alpha}{ }^{X}} \sum_{i j l} A_{i j \alpha l} \bar{f}_{i}^{X} \bar{f}_{j}^{A}-\frac{\partial \ln \bar{f}_{\alpha}{ }^{X}}{\partial t}\right]+\frac{\partial^{2} P_{1}\left(x_{\alpha}, t\right)}{\partial x_{\alpha}{ }^{2}} \\
& \times\left[2 \sum_{i j l} A_{i j \alpha l} \bar{f}_{i}^{X} \bar{f}_{j}^{A}-\frac{1}{2} \frac{\partial \bar{f}_{\alpha}{ }^{X}}{\partial t}\right]
\end{aligned}
$$

and a similar equation for $P_{1}\left(y_{\beta}, t\right)$. The fluctuations $x_{\alpha}$, $y_{\beta}$ have been defined as in Eq. (3.6). Strictly speaking, Eq. (5.2) is coupled to the equation for $P_{1}\left(y_{\beta}, t\right)$ through the average values $f^{X}, f^{Y}$, which must satisfy the selfconsistency conditions

$$
\begin{aligned}
& \tilde{f}_{\alpha}^{X}=\sum_{\left\{f^{X}\right\},\left\{f^{Y}\right\}} f_{\alpha}^{X} P\left(\left\{f^{X}\right\},\left\{f^{Y}\right\}, t\right) \\
& \tilde{f}_{\beta}^{Y}=\sum_{\left\{f^{X}\right\},\left\{f^{Y}\right\}} f_{\beta}^{Y} P\left(\left\{f^{X}\right\},\left\{f^{Y}\right\}, t\right) .
\end{aligned}
$$

To a first approximation (consistent with the FokkerPlanck limit), one is allowed to identify the $f_{s}$ with the macroscopic averages appearing in the Botzmann Eq. (5.1). It follows that Eq. (5.2) admit steady-state solutions of the form (3.7) and that the steady reference state $\tilde{f}_{\alpha}{ }^{X}, \vec{f}_{\beta}{ }^{Y}$ is always stable with respect to small thermal fluctuations. Similarly, the extended Einstein relation (3.8) is valid in the domain of small fluctuations (4). One has, therefore, an a posteriori justification, in the 
domain of small fluctuations, of the results obtained by Kerner (16) and Montroll et al. (18) who postulated relations implying the validity of a canonical distribution of fluctuations.

In conclusion, we see that in the Volterra-Lotka model the analysis based on the birth and death type of formalism is not compatible with the more complete phase-space description. By comparing the two master equations, we see that the inadequacy of the usual stochastic treatment is due to an incorrect account of the internal states.

We have not yet analyzed completely the behavior of large fluctuations of macroscopic size. It appears from a preliminary study (4) that such fluctuations are always time-dependent and may drive the system, for sufficiently long times, to a new regime far from the initial reference state.

\section{CONCLUDING REMARKS. "GENERALIZED TURBULENCE"}

We have shown that in nonlinear systems far from equilibrium, it is generally necessary to adopt a phasespace stochastic description in order to describe correctly the distribution of thermal fluctuations around steady nonequilibrium states. As a consequence, for systems in a local equilibrium regime, small fluctuations always behave according to the generalized Einstein relations (1.1) or (3.7), (3.8).

While this result is quite natural for models of the type discussed in Secs. $\mathscr{Z}$ and 3 , it may appear contradictory when applied to the Volterra--Lotka model or to any system which is at or slightly beyond a state of marginal stability. We believe that the resolution of this apparent paradox, which is related to the very nature of the onset of oscillations and instabilities, may be the following. The lack of asymptotic stability observed in nonlinear dissipative systems (chemical instabilities, etc.) is a purely macroscopic phenomenon that has no molecular counterpart as long as the system (including fluctuations) is maintained in a local equilibrium regime. Thus, it is reasonable to expect that systems undergoing such macroscopic instabilities cannot evolve from a given macroscopic reference state by a mechanism of thermal fluctuations of usual size (i.e., very small). This explains why in the phase-space description of Secs. 3 and 5 small fluctuations are damped. Again, the difference with instabilities in the velocity distribution mentioned in the introduction should be emphasized.

A change in the macroscopic state of a system in a local equilibrium regime can therefore arise only from a mechanism of large thermal fluctuations of macroscopic size. Macroscopic instabilities seem, thus, to bear some analogies with first-order phase transitions.

In order to substantiate this conjecture, it would be necessary to solve the complete master equations in phase space for model (4.3) in a way that takes into account, self-consistently, the simultaneous evolution of the fluctuations and of the macroscopic state. One must study the time-dependent solutions of the master equation for arbitrary fluctuations, imposing at each stage the self-consistency conditions (5.3) for any macroscopic state. This study, which should provide such information as, e.g., the critical size of fluctuations beyond which the system starts to evolve, and the time required for the formation of this evolving mode, is presently in progress.

In conclusion, the separation between macroscopic behavior and fluctuations is related to the distance from thermodynamic equilibrium. In a far from equilibrium regime, corresponding to the onset of oscillations or instabilities, this separation is not possible in general and the evolution of the average values depends explicitly on the fluctuations. The situation brings to mind the familiar phenomenon of fluid dynamics arising beyond instability of the laminar flow and may be appropriately called "generalized turbulence".

We gratefully acknowledge that a first stage of this investigation was done in collaboration with Dr. A. Babloyantz. We are indebted to Prof. M. Kac for stimulating discussions and fruitful suggestions. I. P. would like to express his appreciation to Drs. A. Butterworth and R. Herman for their interest in this work and for stimulating discussions during his stay at the General Motors Research Laboratories.

1. Glansdorff, P., and I. Prigogine, Thermodynamics of Structure, Stability and Fluctuations (Wiley-Interscience, New York, 1971).

2. Balescu, R., Statistical Mechanics of Charged Particles (Wiley-Interscience, New York, 1963).

3. Graham, R., and H. Haken, Phys. Lett., 29A, 530 (1969).

4. Nicolis, G. (submitted to) J. Stat. Phys. An expanded version of the present work, together with a detailed discussion of the biological applications, was presented at the 3rd International Symposium Theoretical Physics and Biology, Versailles (June, 1971).

5. Onsager, L., and 'S. Machlup, Phys. Rev., 91, 1505 (1953); Landau, L. D., and E. M. Lifshitz, Statistical Physics (Pergamon Press, London, 1959).

6. Landau, L. D., and E. M. Lifshitz, Fluid Mechanics (Pergamon Press, Oxford, 1959); Wax, N., ed., Selected Papers on Noise and Stochastic Processes (Dover Publications, New York, 1954).

7. McQuarrie, D., Suppl. Rev. Ser. Appl. Probab. (Methuen and Co., London, 1967); van Kampen, N. G., Advan. Chem. Phys., 15, 65 (1969).

8. Kac, M., Probability and Related Topics in Physical Sciences (Interscience Publishers, New York, 1959).

9. Callen, H. B., Nonequilibrium Thermodynamics, Variational Techniques and Stability, ed. R. Donnelly, R. Herman, and I. Prigogine (University of Chicago Press, Chicago, Ill., 1965).

10. Prigogine, I., and G. Mayer, Bull. Cl. Sci. Acad. Roy. Belg., 41, 22 (1955); Lax, M., Rev. Mod. Phys., 32, 25 (1960); Schlögl, F., Z. Physik 244, 199 (1971).

11. Gantsevich, S. V., V. Gurevich, and R. Katilius, Sov. Phys. JETP, 32, 291 (1971).

12. Nicolis, G., and A. Babloyantz, J. Chem. Phys., 51, 2632 (1969); Hawkins, R., and S. A. Rice, J. Theor. Biol., 30, 579 (1971).

13. Ross, J., and P. Mazur, J. Chem. Phys., 35, 19 (1961).

14. Siegert, A. J. F., Phys. Rev., 76, 1708 (1949).

15. Volterra, V., Leçons sur la Théorie Mathématique de la lutte pour la vie (Gauthier-Villars, Paris, 1931).

16. Kerner, E. H., Bull. Math. Biophys., 19, 121 (1957); 21, 217 (1959)

17. Lefever, R., G. Nicolis, and I. Prigogine, J. Chem. Phys., 47, 1045 (1967).

18. Montroll, E., Rev. Mod. Phys., in press. 